\title{
Is Mammography Useful in Screening for Local Recurrences in Patients With TRAM Flap Breast Reconstruction After Mastectomy for Multifocal DCIS?
}

\author{
A. P. Salas, MD, Mark A. Helvie, MD, Edwin G. Wilkins, MD, Harold A. Oberman, MD, \\ Peter W. Possert, MD, Alan M. Yahanda, MD, and Alfred E. Chang, MD
}

\begin{abstract}
Background: Skin-sparing mastectomy with immediate transverse rectus abdominis musculocutaneous (TRAM) flap reconstruction is being used more often for the treatment of breast cancer. Mammography is not used routinely to evaluate TRAM flaps in women who have undergone mastectomy. We have identified the potential value of its use in selected patients.

Methods and Results: We report on four women who manifested local recurrences in TRAM flaps after initial treatment for ductal carcinoma in situ (DCIS) or DCIS with microinvasion undergoing skin-sparing mastectomy and immediate reconstruction. All four patients presented with extensive, high-grade, multifocal DCIS that precluded breast conservation. Three of four mastectomy specimens demonstrated tumor close to the surgical margin. Three of the four recurrences were detected by physical examination; the remaining local recurrence was documented by screening mammography. The recurrences had features suggestive of malignancy on mammography.

Conclusion: We conclude that all patients undergoing mastectomy and TRAM reconstruction for extensive, multifocal DCIS should undergo regular routine mammography of the reconstructed breast. Our experience with this subgroup of patients raises concern about the value of skin-sparing mastectomy with immediate reconstruction for therapy. Adjuvant radiation therapy should be recommended for those patients with negative but close surgical margins.
\end{abstract}

Key Words: Mammography-Breast cancer-Local recurrence-Ductal carcinoma in situSkin-sparing mastectomy.

Breast reconstruction has been demonstrated to be safe and effective after surgical removal of the breast in properly selected patients with breast cancer. ${ }^{1-4}$ Reconstruction minimizes the deformity and the psychological impact of a mastectomy. ${ }^{5,6}$ The advantages of reconstruction with autologous tissue are that it affords a superior cosmetic result $^{7}$ and is particularly useful in patients who have failed breast conservation therapy and have an irradiated chest wall. Because the timing of reconstruction cannot be expected to compromise the primary opera-

Received October 3, 1997; accepted January 22, 1998.

From the Division of Surgical Oncology (APS, AMY, AEC), and the Plastic Surgery Section (EGW), Department of Surgery, and the Departments of Radiology (MAH), Pathology (HAO), and Radiation Oncology (PWP), University of Michigan Medical Center, Ann Arbor, Michigan.

Address correspondence and reprint requests to: Alfred E. Chang, MD, University of Michigan Medical Center, 1500 E. Medical Center Dr., Ann Arbor, MI 48109-0932. tion, alter survival, or interfere with the detection and treatment of tumor recurrence, ${ }^{1,8}$ immediate reconstruction recently has gained in popularity. The trend toward use of autologous tissue has increased because of the perceived health risks associated with silicone implants. ${ }^{9}$ In addition, skin-sparing mastectomy is being employed more often to improve the cosmetic results of reconstruction. ${ }^{10}$ As a result of the increased use of TRAM flap reconstruction, follow-up and management of local recurrence in the area of the flap has become a more urgent and challenging issue.

Most local recurrences appear in the residual skin and subcutaneous tissues in the area of the mastectomy ${ }^{11-13}$ and may be attributed to residual breast tissue, tumor seeding at the time of surgery, or persistence of tumor in the operative field and lymphatics. ${ }^{14}$ With local recurrences occurring anterior to the pectoralis major muscle, reconstructions involving the placement of a prosthesis 
do not significantly interfere with follow-up efforts because of their subpectoral location. Myocutaneous flap reconstructions, however, occupy the surgical site and give rise to the possibility that the flap may mask locally recurrent disease because of its normal tissue texture, and may lead to a delay in diagnosis. Retrospective studies, however, have reported that the detection of locoregional recurrences in patients undergoing immediate myocutaneous flap reconstruction after mastectomy was not delayed nor was treatment compromised. ${ }^{1,15-18}$

Issues regarding follow-up and management of these patients are still unclear and are further complicated by concerns over issues of cost containment in an environment of fiscal concern. Local recurrences in reconstructed breasts are relatively rare. Because most recurrences occur in the skin and subcutaneous tissues, ${ }^{11-13}$ they usually can be detected on physical examination. Therefore, currently accepted follow-up for patients with myocutaneous flap reconstruction does not involve the use of routine mammography for the detection of local recurrence. Moreover, the routine use of mammography can lead to the detection of flap abnormalities other than recurrent cancer (i.e., fat necrosis) which easily could be mistaken for a recurrence, resulting in unnecessary biopsy procedures. ${ }^{19-21}$

In this report, we review four cases of local recurrences in patients who underwent skin-sparing mastectomy with TRAM flap reconstruction. Characteristic mammographic changes consistent with malignant findings are described in three of the four cases. All of the patients presented initially with extensive, high-grade DCIS and represent a subgroup of patients who have a higher risk of local recurrence. We believe that these patients should undergo routine mammography of their TRAM flaps to identify local recurrences. Moreover, we recommend that patients with negative but close $(<1$ $\mathrm{mm}$ ) surgical margins have adjuvant radiation to reduce their risk of local recurrence.

\section{METHODS}

The University of Michigan Cancer Center is a National Cancer Institute-supported comprehensive cancer center. All newly diagnosed breast cancer patients (excluding stage IV patients) are evaluated in the multidisciplinary Breast Care Center (BCC), which has been operational since 1984. The BCC convenes weekly, and patients are seen by all the relevant disciplines in one clinic setting. A tumor board meets the same day to review all new patients, and treatment options are dis- cussed. Final recommendations are conveyed to the patients the same day they are seen. The BCC evaluates approximately 380 new patients each year.

Recommendations for mastectomy versus breast conservation are made by consensus among members of the BCC tumor board. If mastectomy is required, the option of immediate breast reconstruction is reviewed with the patient. The type of breast reconstruction is determined by the patient in consultation with the plastic surgeon in the BCC.

Skin-sparing mastectomies are performed to obtain optimal cosmetic results. Skin-sparing mastectomies involve removal of all gross breast tissue, including the nipple and areola (along with axillary contents if an axillary dissection is performed). Previous biopsy incisions are removed, either in continuity with the nipple-areolar complex or separately. To optimize reconstruction, maximal preservation of the native skin envelope is achieved, and the inframammary fold is kept intact. The thickness of the mastectomy skin flaps is equivalent to that of mastectomies performed without subsequent reconstruction. The evaluation of the reconstructed breast has not involved routine mammography.

Four women were identified who had recurrent carcinoma in their TRAM flaps following mastectomy for a biopsy diagnosis of DCIS. Initial preoperative mammograms and follow-up mammograms were reviewed. Similarly, the microscopic findings of the initial biopsy and mastectomy were reviewed, and were compared with sections from the recurrent neoplasm.

\section{CASE REPORTS}

We report four cases of local recurrence of carcinoma in patients who had TRAM flap reconstruction following total mastectomy for DCIS. Included in these case studies are the mammographic findings and the approach used in the management of each patient. Table 1 summarizes these cases.

\section{Case 1}

\section{Clinical Summary}

This 48-year-old woman underwent a right modified radical mastectomy and a left prophylactic total mastectomy with immediate bilateral TRAM flap reconstruction following a biopsy diagnosis of right-sided multifocal DCIS in August 1994. The prophylactic left mastectomy was performed at the patient's request. The breast reconstruction consisted of a right free TRAM flap and a left pedicle TRAM flap. She received no adjuvant 
TABLE 1. Summary of cases

\begin{tabular}{|c|c|c|c|c|c|}
\hline Case & $\begin{array}{c}\text { Age at } \\
\text { diagnosis }(y)\end{array}$ & Pathology & $\begin{array}{l}\text { Time to } \\
\text { recurrence }\end{array}$ & $\begin{array}{l}\text { Recurrence } \\
\text { palpable }\end{array}$ & $\begin{array}{l}\text { Treatment of } \\
\text { recurrences }\end{array}$ \\
\hline 1 & 48 & Multifocal DCIS & $1 \mathrm{y}, 9 \mathrm{~m}$ & Yes & $\begin{array}{l}\text { Resection of TRAM, } \\
\text { chemotherapy and } \\
\text { radiation therapy }\end{array}$ \\
\hline 2 & 47 & Multifocal DCIS & $3 y$ & Yes & $\begin{array}{l}\text { Lumpectomy, axillary lymph } \\
\text { node dissection; } \\
\text { chemotherapy and } \\
\text { radiation therapy }\end{array}$ \\
\hline 3 & 37 & $\begin{array}{l}\text { Multifocal DCIS with } \\
\text { focus of microinvasion }\end{array}$ & $2 \mathrm{y}, 3 \mathrm{~m}$ & No & $\begin{array}{l}\text { Resection of TRAM flap, } \\
\text { axillary dissection; } \\
\text { chemotherapy and } \\
\text { radiation therapy }\end{array}$ \\
\hline 4 & 42 & $\begin{array}{l}\text { Multifocal DCIS with } \\
\text { focus of microinvasion }\end{array}$ & $4 \mathrm{y}, 8 \mathrm{~m}$ & Yes & $\begin{array}{l}\text { Lumpectomy, axillary lymph } \\
\text { node dissection; radiation } \\
\text { therapy }\end{array}$ \\
\hline
\end{tabular}

therapy. In March 1995, she had bilateral nipple-areolar reconstruction. Two months later she developed firmness of the reconstructed right breast and some red, nodular skin lesions in the area of the scar around her neo-areola. A biopsy of the skin lesion revealed invasive mucinous carcinoma with dermal angiolymphatic invasion. Mammogram of the right reconstructed breast revealed a $4 \times$ $3 \mathrm{~cm}$ area of pleomorphic calcifications and soft tissue mass that were highly suspicious for malignancy (Fig. 1). She underwent a metastatic work-up that included CT scans of the chest, abdomen, and pelvis, which proved negative. The patient then was treated with resection of the right TRAM flap. The excised specimen contained invasive carcinoma. She subsequently has been treated with chemotherapy and chest wall irradiation and at this time is without evidence of recurrent disease.

\section{Pathologic Findings}

The original breast biopsy contained multifocal DCIS of high nuclear grade, and the subsequent mastectomy specimen also had multiple foci of DCIS, which ex-

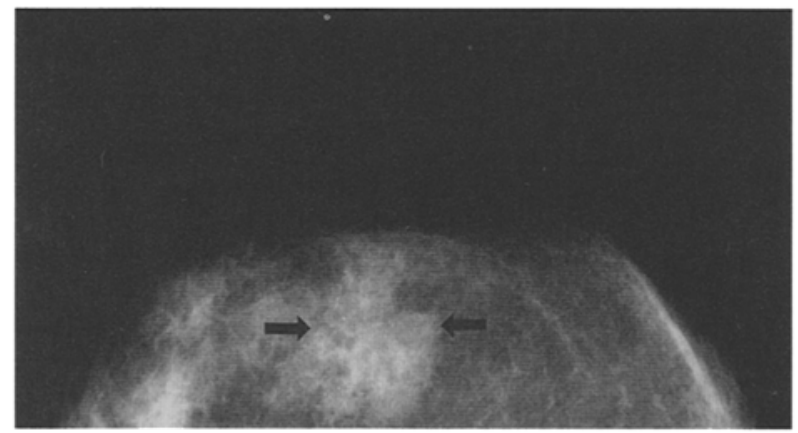

FIG. 1. Craniocaudal mammogram demonstrates a $4 \times 3 \mathrm{~cm}$ area of pleomorphic calcifications with associated soft tissue density in the TRAM flap (arrows). Pathology revealed invasive ductal carcinoma. tended to within $1 \mathrm{~mm}$ of the inked margin of surgical excision. The neoplastic ducts had predominant solid and comedonecrosis patterns of growth.

Invasive ductal carcinoma was present in the May 1995 biopsy and in the subsequent mastectomy. The predominant growth pattern was mucinous carcinoma, representing approximately $90 \%$ of the neoplasm; in contrast to the usual mucinous carcinoma, however, this neoplasm had a high histologic grade and manifested invasion of peritumoral and dermal lymphatics. The neoplasm measured $4 \mathrm{~cm}$ in greatest dimension, was circumscribed, and was surrounded by foci of high nuclear grade DCIS. Carcinoma involved the margins of surgical excision.

\section{Case 2}

\section{Clinical Summary}

This patient was 47 years old when she underwent a left total mastectomy with immediate free TRAM flap reconstruction for multifocal DCIS in August 1993. Three years later she noted a palpable nodule in the superior aspect of her flap, located beneath the preserved breast skin flap raised during the initial mastectomy. Mammogram of the left TRAM flap revealed a highly suspicious, high-density mass $1 \mathrm{~cm}$ in diameter, with spiculated margins and associated pleomorphic microcalcifications in the area of the palpable abnormality (Fig. 2). Fine-needle aspiration cytologic study of the nodule was positive for carcinoma. A metastatic workup, including CT scans of the chest and abdomen, was negative. Wide excision of the involved area was performed, with a left axillary lymph node dissection, in August 1996. The re-excision lumpectomy contained no residual tumor; the axillary lymph node dissection, how- 

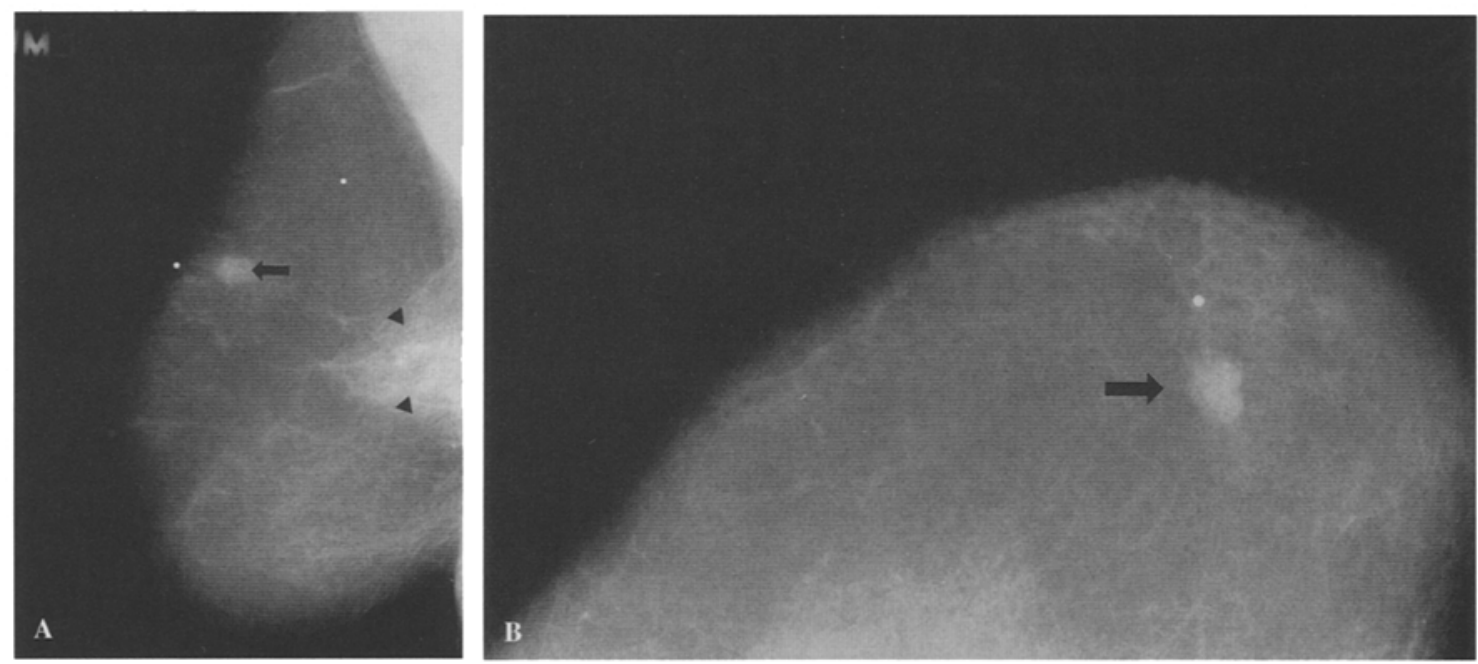

FIG. 2. Mediolateral oblique (A) and craniocaudal (B) mammogram of TRAM flap demonstrates a 1-cm mass with spiculated borders with associated microcalcifications highly suspicious for carcinoma (arrow). Invasive ductal carcinoma was found at biopsy. The triangular-shaped soft tissue (arrowheads) density noted posteriorly on the mediolateral oblique view (A) represents the rectus musculature.

ever, revealed that one of twelve lymph nodes contained metastatic carcinoma. The patient went on to receive adjuvant chemotherapy and radiation therapy and is free of recurrence as of this date.

\section{Pathologic Findings}

The initial biopsy contained multifocal DCIS of high nuclear grade, and the subsequent mastectomy specimen also contained extensive mutifocal DCIS of similar grade. Although the surgical margins were negative, the neoplasm extended to within $1 \mathrm{~mm}$ of the surgical margin of the mastectomy.

The August 1996 lumpectomy specimen contained multiple foci of high nuclear grade DCIS. Moreover, there was an adjacent area of invasive ductal carcinoma of high histologic grade. It measured $1.3 \mathrm{~cm}$ in greatest dimension.

\section{Case 3}

\section{Clinical Summary}

This patient was 37 years old when she underwent a left total mastectomy and a right prophylactic total mastectomy with immediate bilateral free TRAM flap reconstruction for extensive multifocal DCIS of the left breast in February 1995. The prophylactic mastectomy was performed at the patient's request. In May 1995, she underwent TRAM flap recontouring and bilateral nippleareolar reconstruction. Two years later, screening mammography ordered by the patient's primary care physician revealed two separate areas of suspicious calcifications in the left reconstructed breast and two small masses $(<5 \mathrm{~mm})$, of which one had spiculated margins and the other was well circumscribed (Fig. 3). These lesions were located several centimeters below the skin and were not palpable on physical examination. Needle localization biopsy of one area of calcification and of the spiculated mass revealed separate foci of small invasive carcinomas. The patient subsequently underwent resection of the involved flap with an axillary dissection. She is currently disease-free after adjuvant chemotherapy and radiation therapy.

\section{Pathologic Findings}

The initial biopsy specimen contained multifocal DCIS. The neoplasm had a high nuclear grade, and the involved cells were of apocrine type and occasionally vacuolated. Solid, papillary, cribriform growth patterns, as well as comedonecrosis, were seen. The following mastectomy also contained multifocal high nuclear grade DCIS. In addition, one section contained a focus of stromal microinvasion (greatest dimension of invasion $<1$ $\mathrm{mm}$ ). The margins of surgical excision were uninvolved by neoplasm.

The 1997 biopsy contained multifocal DCIS of high nuclear grade, similar to the neoplasm initially seen. The TRAM flap was resected, and and was found to contain not only multifocal DCIS but also three foci of invasive ductal carcinoma. The largest of these measured $6 \mathrm{~mm}$ in diameter; the others were $1-\mathrm{mm}$ lesions. All three had a high histologic grade. A tumor embolus was noted in a lymphatic channel of one axillary tail lymph node; no lymph node parenchymal involvement was found in seven resected nodes, however. 

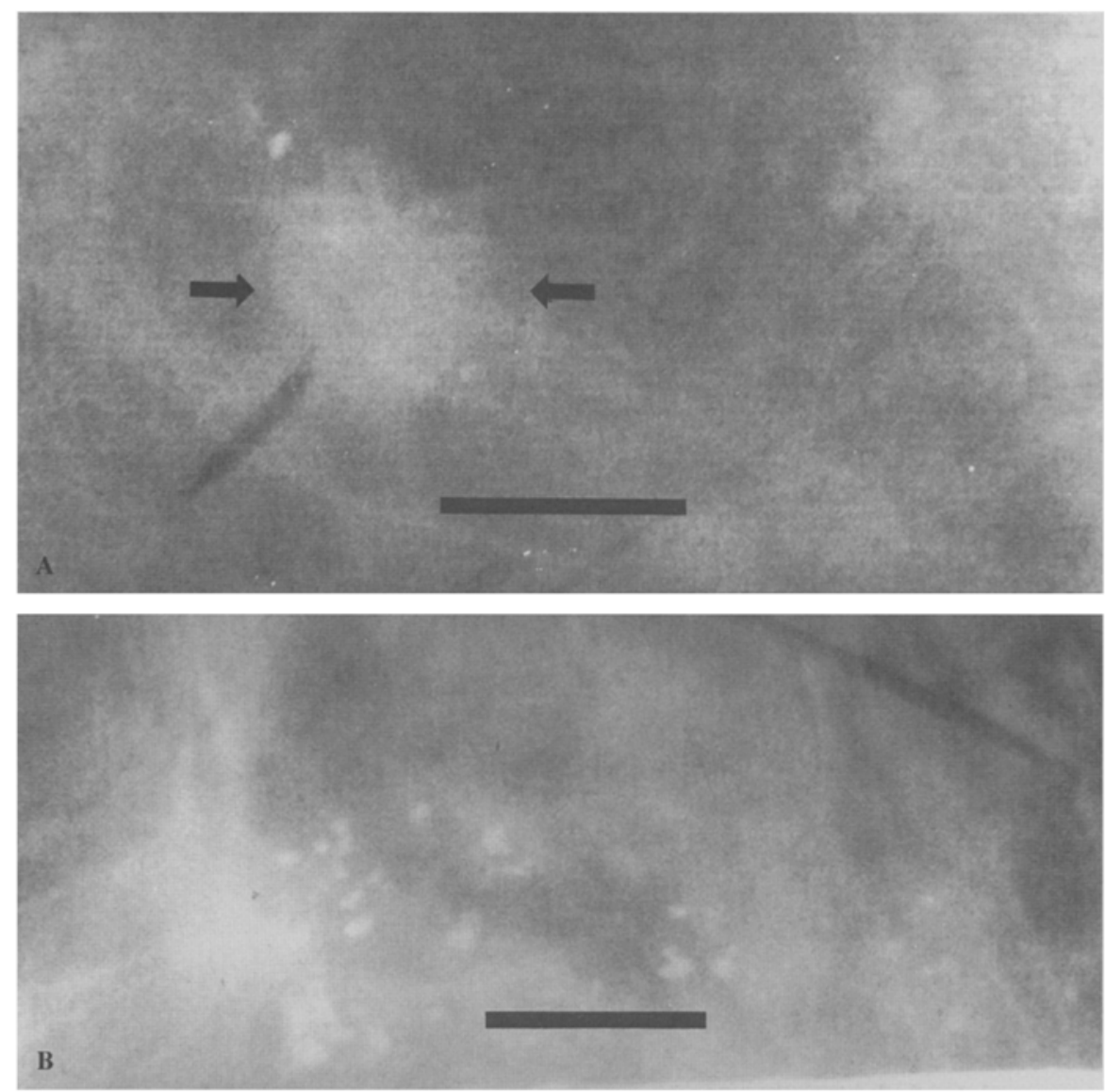

FIG. 3. Close-up mammographic views from a patient with multicentric breast cancer recurrence discovered solely by screening tramogram. (A) A 5-mm irregular marginated mass (arrows) with adjacent calcification located in the upper outer quadrant of the TRAM. (B) Pleomorphic calcifications with soft tissue density noted in the upper inner quadrant $9 \mathrm{~cm}$ from the lesion shown in A. Histology demonstrated invasive ductal carcinoma with highgrade DCIS (black bar $=5 \mathrm{~mm}$ ).

\section{Case 4}

\section{Clinical Summary}

This 42-year-old woman underwent a right total mastectomy with an immediate free TRAM flap reconstruction in September 1992. The flap was recontoured and a nipple-areola complex was constructed in March 1995. One year later invasive ductal carcinoma of the left breast was diagnosed, and the patient was treated with a modified radical mastectomy and immediate reconstruction at another institution. Axillary lymph node dissection of that specimen lacked metastatic carcinoma, and she received 6 months of postoperative adjuvant chemotherapy followed by tamoxifen. In May 1997, she presented with a small nodule in her right TRAM flap, which was biopsied and found to be an invasive ductal carcinoma with a noninvasive component, extending to the surgical margin. A subsequent mammogram of the residual TRAM flap did not reveal any other abnormality. She was treated with a reexcisional lumpectomy and axillary lymph node dissection. The latter revealed residual DCIS in the lumpectomy site and absence of lymph node metastases. She then was treated with irradiation to the residual TRAM flap.

\section{Pathologic Findings}

The right breast biopsy performed in July 1994 contained multifocal, high nuclear grade DCIS with a predominant solid growth pattern and with focal stromal microinvasion. Extensive DCIS was present in the right mastectomy specimen taken in September 1994. The DCIS had a focally papillary growth pattern and involved ectatic ducts, which extended to within $1 \mathrm{~mm}$ of a surgical margin of excision. Stromal microinvasion also was present in this material.

The biopsy specimen taken in May 1997 contained a 1.6- $\mathrm{cm}$ circumscribed focus of invasive ductal carcinoma of high histologic grade. The biopsy performed the following month also found multiple foci of DCIS of high nuclear grade with focal microinvasion of stroma.

\section{DISCUSSION}

As a result of the growing popularity and prevalence of TRAM flap breast reconstruction, it has become in- 
creasingly important to define the approach used in follow-up of patients choosing this alternative. Mammography usually has not been included as part of routine follow-up unless an abnormality is detected on physical examination. Many researchers consider mammography to be of little benefit for oncologic follow-up in reconstructed patients. ${ }^{22}$ Moreover, breast imaging can lead to findings that give false-positive results, including such abnormalities as fat necrosis (commonly seen in the early postoperative period), oil cysts (which can be calcified), and scar tissue. ${ }^{19-21}$ Because recurrent carcinoma also can occur in the early postoperative period, equivocal findings cannot simply be overlooked. Prospective studies have not adequately addressed the routine use of mammography in the surveillance of myocutaneous flaps. Because flap recurrences are not common, the cost-benefit ratio of detecting nonpalpable recurrences may be low.

Certain representative mammographic characteristics are common to most autologous reconstructions. Myocutaneous flaps have a relatively uniform fatty appearance, but lack the usual organization and ductal structures of the normal breast. They also lack the typical vascular and connective tissue markings seen on normal mammograms. The rectus muscle often can be seen anterior to the pectoralis major muscle on the mediolateral oblique view. Surgical scars also are seen routinely, although they vary considerably between patients. Masses identified in autologous flaps can represent fat necrosis, scars, suture granulomas, chest wall cysts, ${ }^{19-21}$ and, most importantly, recurrent cancer. We have noted that the typical radiographic appearance of malignant microcalcifications and masses can be associated with either recurrent malignancy or new primary cancers in mammograms of the TRAM flaps.

The cases we have described in this report are striking in their similarity of presentation and define a subgroup of patients who could benefit from routine postoperative screening mammographic studies of the TRAM flap, which we have named "tramograms." All four of our patients initially presented with extensive multifocal DCIS, two of them with microinvasive disease. In all four cases, the DCIS was found to be high grade. Three of the four patients had tumor close (i.e., $<1 \mathrm{~mm}$ ) to the surgical margin. Historically, patients with all forms of DCIS fare well with conventional total mastectomy. In a review by Delaney et al., 1707 patients summarized from 9 published reports were treated for DCIS with mastectomy alone, with a median follow-up of $>60$ months. ${ }^{23}$ There were ten local recurrences, representing $1.4 \%$ of the total group, with five of the ten (50\%) being invasive recurrences. The known risk factors for local recurrence after surgical resection (mastectomy as well as lumpectomy) of DCIS have been reported to be close or involved margins and the presence of necrosis or highgrade tumors. ${ }^{23-28}$ The relatively high incidence of invasive disease (ranging from $54 \%$ to $82 \%$ ) $^{23,29-31}$ associated with local recurrences after surgical therapy for DCIS emphasizes the importance of careful followup in patients with these risk factors. We believe that radiographic surveillance of TRAM flaps for patients who had mastectomies for multifocal DCIS is mandatory and have implemented this screening practice at our institution. This screening should be performed for the lifetime of the patient, because recurrences of low-grade DCIS may evolve over a longer interval.

Our cases bring up concerns regarding the use of skinsparing mastectomy with immediate TRAM flap reconstruction in patients with extensive DCIS. This issue has not been examined in any large series to date. A retrospective study by Kroll et al. evaluated 104 patients with early-stage invasive cancer (excluding DCIS) treated by skin-sparing mastectomies and reconstruction, and found no increase in local or systemic recurrences compared to cases treated by conventional mastectomy. ${ }^{32}$ Carlson et al. evaluated 327 patients with all stages (stages 0 through IV) of breast cancer treated with skin-sparing mastectomies and did not identify an increased incidence of local recurrence in patients with invasive cancers undergoing this procedure. ${ }^{33}$ In their series, no local recurrences were reported in stage 0 patients; the total number of patients with DCIS in this group was not described, however, nor was there an assessment of those patients with focal versus multifocal disease. Obtaining negative surgical margins with skin-sparing mastectomies for patients with extensive multifocal DCIS may be more problematic than it is in patients with focal DCIS. Although all four of our cases were found to have negative surgical margins, the margins were close in three of them. From this experience, we would advocate the use of adjuvant radiation therapy when close margins are identified. The relative risk of local recurrences in this disease setting in patients undergoing skin-sparing mastectomy and immediate reconstruction must be evaluated in the context of a larger data set, which is beyond the scope of this report.

The management of isolated local recurrences in TRAM flaps in patients who presented initially with extensive DCIS has not been well documented. All four of our patients recurred within a 5-year period with invasive ductal carcinomas. None of them presented with metastatic stage IV disease. Two were treated with resection of the TRAM flap followed by systemic chemotherapy and radiation to the chest wall. Two were treated with lumpectomy and axillary lymph node dissection. We en- 
countered no problems associated with flap viability in the two patients who required an axillary lymph node dissection. One of the two patients had lymph node metastases and received chemotherapy as well as radiation therapy to the residual TRAM flap; the other was node negative and received adjuvant radiation only. With the very short follow-up period after salvage treatment in our patients it is too early to determine the ultimate overall survival in these patients; however, all are disease-free at the time of this report.

There is currently no conclusive evidence supporting the use of mammography for routine screening of all patients with flap reconstructions. However, many examinations are being performed by physicians, either in response to patient demand or as a result of anecdotal experience. Other anecdotal reports have described the detection by screening mammography of nonpalpable, locally recurrent cancer in reconstructed breasts postmastectomy. ${ }^{34,35}$ Whether recurrences in TRAM flaps detected earlier with mammography can be treated more successfully than those detected by routine physical examination is unknown and would probably be difficult to determine, based on the infrequent occurrence of such recurrences. Sparing the TRAM flap with early detection of a local recurrence may represent a secondary benefit of routine tramography. We have instituted a program to screen all of our breast cancer patients who have TRAM flaps to determine prospectively the value of this approach and to characterize the natural history of radiologic findings in these patients.

\section{REFERENCES}

1. Johnson CH, van Heerden JA, Donohue JH, Martin JK Jr, Jackson IT, Ilstrup DM. Oncological aspects of immediate breast reconstruction following mastectomy for malignancy. Arch Surg 1989; 124:819-24.

2. Kroll SS, Ames F, Singletary SE, Schusterman MA. The oncologic risks of skin preservation at mastectomy when combined with immediate reconstruction of the breast. Surg Gynecol Obstet 1991; $172: 17-20$

3. Noguchi M, Fukushima W, Ohta N, et al. Oncological aspect of immediate breast reconstruction in mastectomy patients. $J$ Surg Oncol 1992;50:241-6.

4. Patel RT, Webster DJ, Mansel RE, Hughes LE. Is immediate postmastectomy reconstruction safe in the long-term. Eur J Surg Oncol $1993 ; 19: 372-5$.

5. Schain WS. Breast reconstruction. Update of psychosocial and pragmatic concerns. Cancer 1991;68:1170-5.

6. Schain WS, Wellisch DK, Pasnau RO, Landsverk J. The sooner the better: a study of psychological factors in women undergoing immediate versus delayed breast reconstruction. Am J Psychiatry $1985 ; 142: 40-6$.

7. Eberlein TJ, Crespo LD, Smith BL, Hergrueter CA, Douville L, Eriksson E. Prospective evaluation of immediate reconstruction after mastectomy. Ann Surg 1993;218:29-36.

8. Noone RB, Murphy JB, Spear SL, Little JW 3d. A 6-year experi- ence with immediate reconstruction after mastectomy for cancer. Plast Reconstr Surg 1985;76:258-69.

9. Schusterman MA, Kroll SS, Reece GP, Miller MJ, Ainslie N, Halabi S, Balch CM. Incidence of autoimmune disease in patients after breast reconstruction with silicone gel implants versus autogenous tissue: a preliminary report. Ann Plast Surg 1993:31:1-6.

10. Elkowitz A, Colen S, Slavin S, Seibert J, Weinstein M, Shaw W. Various methods of breast reconstruction after mastectomy: an economic comparison. Plast Reconstr Surg 1993;92:77-83.

11. Donegan WL, Perez-Mesa CM, Watson FR. A biostatistical study of locally recurrent breast carcinoma. Surg Gynecol Obstet 1966; 122:529-40.

12. Gilliland $\mathrm{MD}$, Larson $\mathrm{DL}$, Copeland $\mathrm{EM}$. Appropriate timing for breast reconstruction. Plast Reconstr Sutrg 1983;72:335-40.

13. Slavin SA, Love SM, Goldwyn RM. Recurrent breast cancer following immediate teconstruction with myocutaneous flaps. Plast Reconstr Surg 1994;93:1191-1207.

14. Auchincloss $\mathrm{H}$. The nature of local recurrence following radical mastectomy. Cancer 1958;11:611.

15. Georgiade GS, Riefkohl R, Cox E, McCarty KS, Seigler HF, Georgiade NG, Snowhite JC. Long-term clinical outcome of immediate reconstruction after mastectomy. Plast Reconstr Surg 1985;76: 415-20.

16. Georgiade GS. Immediate reconstruction of the breast following modified radical mastectomy for carcinoma of the breast. Clin Plast Surg 1984;11:383-8.

17. Frazier TG, Noone RB. An objective analysis of immediate simultaneous reconstruction in the treatment of primary carcinoma of the breast. Cancer 1985;55:1202-5.

18. Webster DJ, Mansel RE, Hughes LE. Immediate reconstruction of the breast after mastectomy. Is it safe?. Cancer 1984;53:1416-9.

19. Mendelson EB. Evaluation of the postoperative breast. Rad Clin North Am 1992;30:107-38.

20. Slavin SA, Local recurrence after mastectomy and breast reconstruction. In: Grotting JC, ed. Re-operative Aesthetic and Reconstructive Plastic Surgery. St. Louis: Quality Medical Publishing, 1995:1185-1206.

21. Slavin SA, Howrigan PJ, Goldwyn RM. Pseudocyst formation after rectus flap breast reconstruction: diagnosis and treatment. Plast Reconstr Surg 1989;83:670-5.

22. Puckett CL. Mammograms of the reconstructed breast. Plast Reconstr Surg 1991;88:482-3.

23. Delaney G, Ung O, Cahill S, Bilous M, Boyages J. Ductal carcinoma in situ. Part 2: Treatment. Aust NZJ Surg 1997;67:157-65.

24. Jacquemier J, Kurtz JM, Amalric R, Brandone H, Ayme Y, Spitalier JM. An assessment of extensive intraductal component as a risk factor for local recurrence after breast-conserving therapy. $\mathrm{Br}$ $J$ Cancer 1990;61:873-6.

25. Fisher ER, Costantino J, Fisher B, Palekar AS, Redmond C, Mamounas E. Pathologic findings from the National Surgical Adjuvant Breast Project (NSABP) Protocol B-17. Intraductal carcinoma (ductal carcinoma in situ). Cancer 1995;756:1310-9.

26. Cutuli B, Teissier E, Piat JM, Janser JC, Renaud R, Rodier JF, Jung GM. Radical surgery and conservative treatment of ductal carcinoma in situ of the breast. Eur J Cancer 1992;28:649-54.

27. Zafrani B, Vielh P, Fourquet A, Mosseri V, Durand JC, Salmon RJ, Vilcoq JR. Conservative treatment of early breast cancer: prognostic value of the ductal in situ component and other pathological variables on local control and survival. Long-term results. Eur $J$ Cancer Clin Oncol 1989;25:1645-50.

28. Silverstein MJ, Lagios MD. Use of predictors of recurrence to plan therapy for DCIS of the breast. Oncology 1997;11:393-406, 409415.

29. Price P, Sinnett HD, Gusterson B, Walsh G, A'Hern RP, McKinna JA. Duct carcinoma in situ: predictors of local recurrence and progression in patients treated by surgery alone. Br J Cancer 1990; 61:869-72.

30. Solin LJ, Recht A, Fourquet A, et al. Ten-year results of breast- 
conserving surgery and definitive irradiation for intraductal carcinoma (ductal carcinoma in situ) of the breast. Cancer 1991;68: 2337-44.

31. Silverstein MJ, Cohlan BF, Gierson ED, et al. Duct carcinoma in situ: 227 cases without microinvasion. Eur J Cancer 1992;28:63044.

32. Kroll SS, Schusterman MA, Tadjalli HE, Singletary SE, Ames FC Risk of recurrence after treatment of early breast cancer with skinsparing mastectomy. Ann Surg Oncol 1997;4:193-7.
33. Carlson GW, Bostwick J 3rd, Styblo TM, Moore B, Bried JT, Murray DR, Wood WC. Skin-sparing mastectomy. Oncologic and reconstructive considerations. Ann Surg 1997;225:570-8.

34. Dowden RV. Mammography after implant breast reconstruction. Plast Reconstr Surg 1991;1:119-121.

35. Mund D, Wolfson P, Gorczyca DP, Fu YS, Love SM, Bassett LW. Mammographically detected recurrent nonpalpable carcinoma developing in a transverse rectus abdominis myocutaneous flap. Cancer 1994;74:2804-7. 